\title{
Adaptive Coding and Modulation Scheme for Ka Band Space Com- munications
}

\author{
Jaeyoon Lee, Dongweon Yoon ${ }^{\dagger}$, and Wooju Lee
}

Department of Electronics and Communications Engineering

Hanyang University, Seoul 133-791, Korea

Rain attenuation can cause a serious problem that an availability of space communication link on Ka band becomes low. To reduce the effect of rain attenuation on the error performance of space communications in Ka band, an adaptive coding and modulation (ACM) scheme is required. In this paper, to achieve a reliable telemetry data transmission, we propose an adaptive coding and modulation level using turbo code recommended by the consultative committee for space data systems (CCSDS) and various modulation methods (QPSK, 8PSK, 4+12 APSK, and 4+12+16 APSK) adopted in the digital video broadcasting-satellite2 (DVB-S2).

Keywords: adaptive coding and modulation, turbo code, amplitude phase shift keying

\section{INTRODUCTION}

In 2007, Korea established the space development plan and showed a positive space exploration plan aiming for the development of a lunar probe until 2020 (Lee 2009). Since a disconnection in a communication link can occur in the communications for space exploration due to the locations of the transmitter and the receiver and/or the movement of heavenly bodies, large capacity data should be transmitted in a given limited time. Thus, a much higher transmission capacity than the conventional one should be secured in the given bandwidth and signal power. The technologies necessarily required for this include use of the high-order modulation with high bandwidth efficiency, application of channel coding method with the error performance near to Shannon Limit, and securing of a wider bandwidth using Ka band frequency. However, in the space communications using Ka band frequency, a rainfall can reduce the link availability by seriously affecting the communication link. To reduce the time percentage and to increase the link avail- ability, operation with enhanced link margin is needed. It can, however, increase the power consumption by the system. Thus, an adaptive coding and modulation (ACM) scheme is required to minimize the power loss by rainfall in the space communications in Ka band. Digital video broadcasting - satellite - second generation (DVB-S2) which provides the satellite broadcasting in Ka band compensates the rain attenuation by setting the ACM levels composed of totally 28 different levels (European Telecommunications Standards Institute [ETSI] 2009).

To continuously transmit large capacity telemetry data with error-free in space communications, channel coding schemes such as turbo code and/or low density parity check (LDPC) code should be used in addition to a bandwidth efficient modulation method. It is well known that turbo code has the near-Shannon Limit error performance and the encoder of turbo code is relatively simpler than that of LDPC code. Consultative Committee for Space Data Systems (CCSDS) has recommended the turbo code as a channel code for space communications in which two convolution encoders with the constraint (c) This is an Open Access article distributed under the terms of the Creative Commons Attribution Non-Commercial License (http://creativecommons.org/licenses/by-nc/3.0/) which permits unrestricted non-commercial use, distribution, and reproduction in any medium, provided the original work is properly cited.
Received February 3, 2010 Accepted March 27, 2010

†Corresponding Author

E-mail: dwyoon@hanyang.ac.kr

Tel:+82-2-2220-0362 Fax: +82-2-2281-9912 
length of 5 and the code rates of $1 / 2,1 / 3,1 / 4$ and $1 / 6$, are parallel concatenated (CCSDS 2003). Amplitude phase shift keying (APSK) is a modulation method by which large capacity data can be transmitted without additional increase of bandwidth, and shows the similar performance to that of rectangular M-ary quadrature amplitude modulation (M-QAM) in a linear AWGN channel. In addition, APSK is an appropriate method for the satellite and space communications using a high power amplifier such as traveling wave tube amplifier (TWTA) and/or solid-state power amplifier (SSPA). Such APSK modulation method can have various types according to the $\mathrm{M}$ levels. 4+12 APSK and 4+12+16 APSK of the various types have been adopted as the standard modulation methods in DVB-S2 in addition to the conventional quadrature phase shift keying (QPSK) and 8PSK (ETSI 2009), and they are also considered as the modulation methods for a high data rate telemetry transmission in CCSDS Experimental Specification (CCSDS 2007).

In this paper, for the reliable telemetry transmission in the space communications in Ka band, we propose the ACM level applying the turbo code recommended by CCSDS and QPSK, 8PSK, 4+12 APSK and 4+12+16 APSK to overcome the performance loss due to rain attenuation. For this purpose, optimum ring ratios for $4+12$ and $4+12+16$ APSK signals are investigated for each coding rate, and the required $E_{\mathrm{b}} / N_{0}$ is obtained for all the modulation methods by analyzing the bit error rate (BER). Finally, we decide the ACM level through the spectral efficiency and the required $E_{\mathrm{b}} / N_{0}$, derive the link margin according to the level, and analyze the time percentage and link availability under the domestic raining conditions.

\section{SYSTEM MODEL AND OPTIMUM RING RATIO}

In this paper, we assumed the ACM system that can reduce the signal attenuation by rainfall in the atmospheric environment to continuously transmit large capacity telemetry data with high efficiency and high reliability. Fig. 1 shows the block diagram of the ACM system that the turbo codes with the coding rates of $1 / 2,1 / 3,1 / 4$, and $1 / 6$, and the modulation methods of QPSK, 8PSK, $4+12$ APSK, and $4+12+16$ APSK are applied. Fig. 2 shows the CCSDS-recommended turbo code. As shown in Fig. 1, the receiver (ground station) measures the signal to interference and noise ratio (SINR) from the received signals and selects the coding rate and the modulation order using SINR. And then, the selected system information is fed back to the transmitter (lunar probe). The transmitter, using the information received from the receiver, alters the coding rate and the modulation method adaptively to the channel conditions, and then, transmits the information data to the receiver.

To apply either $4+12$ APSK or $4+12+16$ APSK, which are considered in Fig. 1, the optimum constellations should first be obtained according to the coding rates of the turbo encoder. The optimum constellations of the modulation methods, 4+12 APSK and 4+12+16 APSK, for a given SNR, or equivalently, for a given spectral efficiency can be obtained by using mutual information or capacity. The mutual information is calculated using the following equation (Costello et al. 1998, De Gaudenzi et al. 2006),

$f(\chi)=I(X ; Y)=\log _{2} M-\frac{1}{M} \sum_{x \in \chi} \mathrm{E}_{n}\left[\log _{2}\left(\sum_{x^{\prime} \in X} \exp \left[-\frac{1}{N_{0}}\left(\left|\sqrt{E_{s}}\left(x-x^{\prime}\right)+n\right|^{2}-|n|^{2}\right)\right]\right)\right]$

where $\chi$ is a transmitted signal set, Es is an average symbol energy and $\mathrm{n}$ is a Gaussian distributed noise random variable with zero mean and variance of $N_{0} / 2$.

Typically $4+12$ APSK has two rings $\left(r_{\mathrm{i}}, i=1,2\right)$, whereas $4+12+16$ APSK has three rings $\left(r_{\mathrm{i}}, i=1,2,3\right)$. Considering the normalized average symbol energy for each modulation method, the optimum ring ratio according the $E_{s} /$ $N_{0}$ and the corresponding mutual information calculated

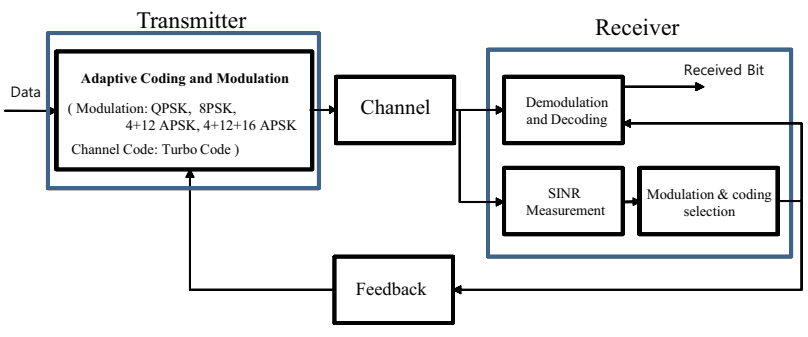

Fig. 1. System model.

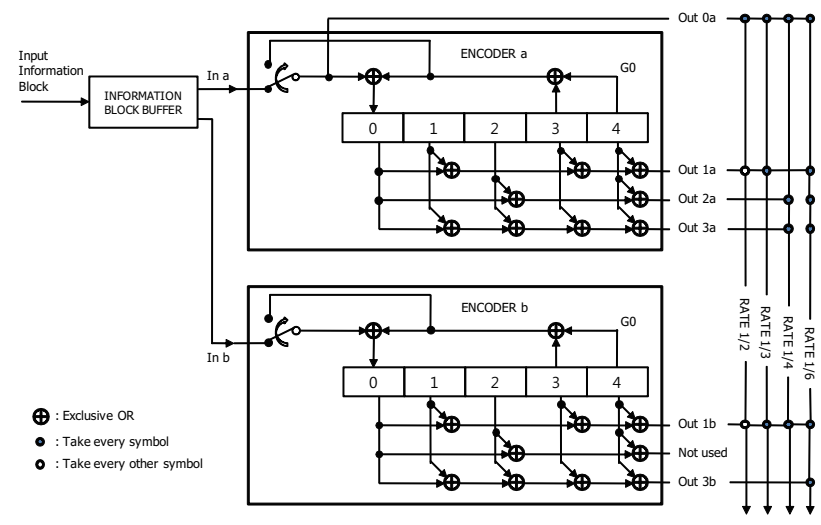

Fig. 2. CCSDS-recommended turbo encoder. 

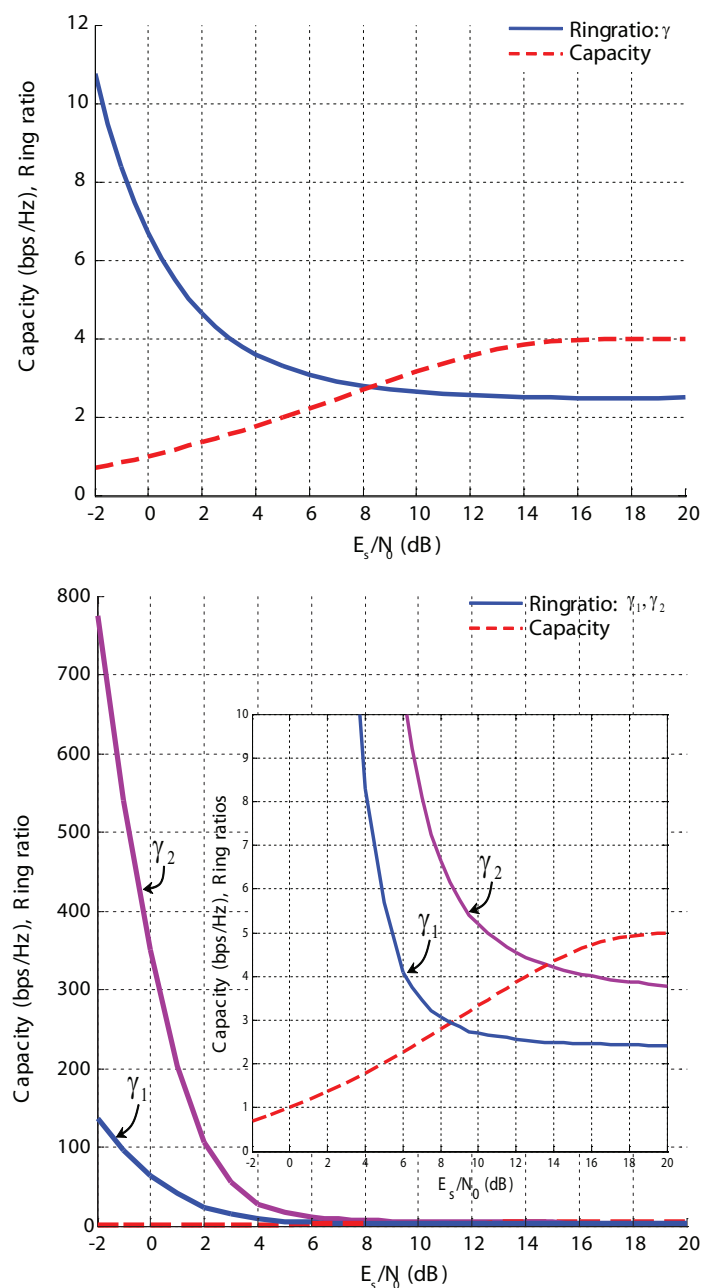

Fig. 3. Mutual information and optimum ring ratio according to $E_{s} / N_{0}$. (a) 4+12 APSK, (b) 4+12+16 APSK.

Table 1. Optimum ring ratios for $4+12$ and $4+12+16$ APSK.

\begin{tabular}{lccc}
\hline $\begin{array}{l}\text { Coding rate } \& \\
\text { modulation }\end{array}$ & $\begin{array}{c}\text { Spectral eff. } \\
(\mathbf{b} / \mathbf{s} / \mathrm{Hz})\end{array}$ & $\begin{array}{c}\text { Ring ratio } \\
\gamma_{1}\left(=\mathbf{r}_{2} / \mathbf{r}_{1}\right)\end{array}$ & $\begin{array}{c}\text { Ring ratio } \\
\gamma_{2}\left(=\mathbf{r}_{3} / \mathbf{r}_{1}\right)\end{array}$ \\
\hline $1 / 2,4+12$ APSK & 2.00 & 3.28 & N/A \\
$1 / 3,4+12$ APSK & 1.33 & 4.71 & N/A \\
$1 / 4,4+12$ APSK & 1.00 & 6.62 & N/A \\
$1 / 6,4+12$ APSK & 0.67 & 11.51 & N/A \\
$1 / 2,4+12+16$ APSK & 2.50 & 3.51 & 8.33 \\
$1 / 3,4+12+16$ APSK & 1.67 & 11.37 & 41.21 \\
$1 / 4,4+12+16$ APSK & 1.25 & 33.60 & 161.20 \\
$1 / 6,4+12+16$ APSK & 0.83 & 99.80 & 563.60
\end{tabular}

APSK: amplitude phase shift keying.

from Eq. (1) are shown in Fig. 3. As shown in Fig. 3, the optimum ring ratio of the uncoded $4+12$ APSK is $\gamma=r_{2} /$ $\mathrm{r}_{1}=2.47$, and the optimum ring ratios of the uncoded
$4+12+16$ APSK are $\gamma_{1}=r_{2} / r_{1}=2.42$ and $\gamma_{2}=r_{3} / r_{1}=3.73$. The optimum ring ratios for each coding rate of turbo code recommended by CCSDS are summarized in Table 1.

\section{ACM FOR A RELIABLE TELEMETRY TRANSMIS- SION}

Rainfall seriously decreases the link availability in Ka band space communications. To increase the link availability, we should increase the link margin over a certain value or use the hierarchical modulation. However, these methods also increase the system power consumption and are slow in responding to rainfall environmental change. One of the best solutions for this impairment is the use of ACM scheme which can minimize the power loss by rainfall. In this section, we provide the ACM level for the reliable telemetry data transmission.

\subsection{ACM level}

Fig. 4 shows the BER curves for turbo coded QPSK, 8PSK, 4+2 APSK, and 4+12+16 APSK with the optimum ring ratios presented in Table 1. Here, the information block size is 8,920 bits, the square-root raised cosine (SRRC) filter is considered as the spectral shaping filter and the roll-off factors of the filter for each modulation method are 0.2 for QPSK and 0.35 for the others. Fig. 5 shows $E_{b} / N_{0}$ required to satisfy the target BER and the capacity for the modulation methods applied in ACM scheme, where we assume that the target BER for reliable communication is $10^{-5}$.

As can be seen from Figs. 4 and 5, the BER performances for the $4+12$ APSK code rates of $1 / 3,1 / 4$ and $1 / 6$ are considerably close to each other. The reason for this is that the coding gain obtained is less than the expected since the minimum distance between the signal points on the inner ring $\left(r_{1}\right)$ is decreased due to the increased ring ratio. The effect caused by the increased ring ratio occurs also in the case of $4+12+16$ APSK. However, the BER performances for the coding rates are not close to each other as much as $4+12$ APSK, since the number of signal points located on the inner ring where the minimum distance reduces the most is considerably less than the total number of signal points. Table 1 shows that the ring ratio of $4+12$ APSK abruptly increases as the coding rate decreases. It also shows that, in 4+12+16 APSK, the ring ratio related to the inner ring increases sharply, while the ratios between the middle ring $\left(r_{2}\right)$ and the outer ring $\left(r_{3}\right)$ do not increase significantly. This indicates that the 
performance degradation due to the increased ring ratio can be more serious in $4+12$ APSK where the ratio of the number of the signal points to the total number of signal points is higher than that in the case of $4+12+16$ APSK.

From summarization of the coding rates and modulation methods shown in Fig. 5 based on the required $E_{b} / N_{0}$ according to each spectrum efficiency, a final ACM level is obtained as shown in Table 2. Here, the link margin is obtained by applying the required $E_{b} / N_{0}$ for each level presented in Table 2 to the down-link model considered in Lee et al. (2010). As in Lee et al. (2010), under the assumption of the system bandwidth of $26 \mathrm{MHz}, E_{b} / N_{0}$ calculated from the link performance parameters such as type of the antenna, the pointing loss and the transmit power for each coding rate and modulation method is given as

$$
E_{\mathrm{b}} / N_{0}=C / N_{0}-R_{\mathrm{b}}
$$

where $C / N_{0}$ which is the carrier power to noise power density ratio is obtained by adding the carrier power to noise power ratio $(C / N)$ to the noise bandwidth $\left(B_{N}\right)$. The $\mathrm{C} / \mathrm{N}$ is obtained as follows:

$$
C / N=P_{R}-P_{N}
$$

In Eq. (3), $P_{R}$ denotes the received power calculated by $P_{R}=G_{T}+P_{T}+G_{R}-L_{T}$, where $G_{T}$ is the transmitter antenna gain, $P_{T}$ is the transmit power, $G_{R}$ is the receiver antenna gain and $L_{T}$ is the sum of the losses by the external factors such as free space loss, atmospheric attenuation, rain attenuation, and so on.

Table 2 shows the link margin derived through the calculated $E_{b} / N_{0}$ obtained by Eq. (2) and the required $E_{b} / N_{0}$ obtained from Fig. 4 for the ACM levels.

\subsection{Application to domestic rain environment}

The rain characteristics can be represented by the rainfall intensity which is precipitation of rainfall per unit hour $(\mathrm{mm} / \mathrm{hr})$. Time percentage is another important physical quantity represented as the percentage of time that specified rainfall intensity is exceeded (Roddy 2006). In Korea, the rainfall intensity varies depending on the regions. For example, when the time percentage is less than $0.01 \%$, the rainfall intensity range is from the minimum 30 to the maximum $55 \mathrm{~mm} / \mathrm{hr}$, depending on the regions (Lee \& Shin 1998). Figs. 6 and 7 show the rain attenuations at $32 \mathrm{GHz}$ frequency in Ka band for the time percentages during one-year period and the four-month maximum rainfall season in the areas, minimum and maximum rainfall regions in Korea, based on the analysis of Lee \& Shin (1998). Also, the figures show range of each ACM level on the time percentage based on the link margin shown in Table 2.

As can be seen from Figs. 6 and 7, if it is assumed that the ACM method is not applied, and the highest coding rate, $1 / 2$, and $4+12+16$ APSK are used, approximately $30.71 \mathrm{~dB}$ of rain attenuation can be overcome. In the case where the link margin is $30.71 \mathrm{~dB}$, during the four-month maximum rainfall season, the time percentages are $0.0738 \%$ in the maximum rainfall region and $0.006385 \%$ in the minimum rainfall region, approximately, and the link availabilities are $99.9262 \%$ and $99.993615 \%$ in the maximum and minimum rainfall regions, respectively. On the other hand, when the ACM scheme applied, the time percentages can be reduced down to about $0.0286 \%$ in the maximum rainfall region and $0.001563 \%$ in the minimum rainfall region, and thus, the link availabilities can be improved up to $99.9714 \%$ and $99.998437 \%$, respectively. Table 3 shows the time percentages in the maximum and minimum rainfall regions for the ACM levels.

From Tables 2 and 3, we see that the application of

Table 2. ACM level.

\begin{tabular}{cccccc}
\hline Level & $\begin{array}{c}\text { Coding rate \& } \\
\text { modulation }\end{array}$ & $\begin{array}{c}\text { Spectral eff. } \\
(\mathbf{b} / \mathbf{s} / \mathbf{H z})\end{array}$ & $\begin{array}{c}\text { Calculated } \\
E_{b} / N_{o}(\mathbf{d B})\end{array}$ & $\begin{array}{c}\text { Required } \\
E_{b} / N_{0}(\mathbf{d B})\end{array}$ & $\begin{array}{c}\text { Link margin } \\
(\mathbf{d B})\end{array}$ \\
\hline 1 & $1 / 6$, QPSK & 0.33 & 46.22 & 0.69 & 45.53 \\
2 & $1 / 4$, QPSK & 0.50 & 44.42 & 0.92 & 43.50 \\
3 & $1 / 4$, QPSK & 0.67 & 43.15 & 1.22 & 41.93 \\
4 & $1 / 2$, QPSK & 1.00 & 41.41 & 1.66 & 39.75 \\
5 & $1 / 2$, QPSK & 1.50 & 39.65 & 3.21 & 36.44 \\
6 & $1 / 2,4+12$ APSK & 2.00 & 38.40 & 4.63 & 33.77 \\
7 & $1 / 2,4+12+16+$ APSK & 2.50 & 37.43 & 6.72 & 30.71 \\
\hline
\end{tabular}

ACM: adaptive coding and modulation, QPSK: quadrature phase shift keying, APSK: amplitude phase shift keying. 


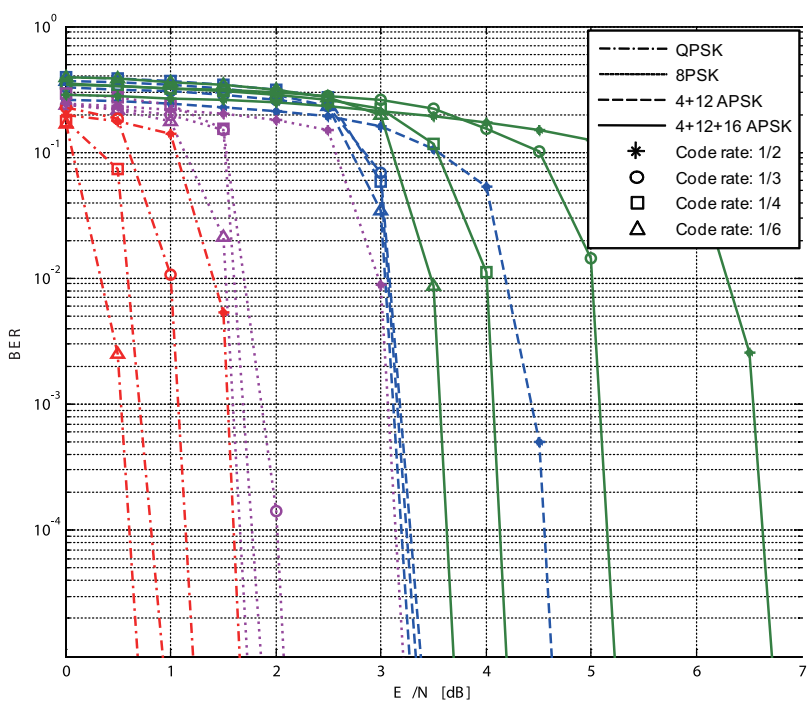

Fig. 4. BER performance for turbo coded QPSK, $8 P S K, 4+12$ APSK, and $4+12+16$ APSK

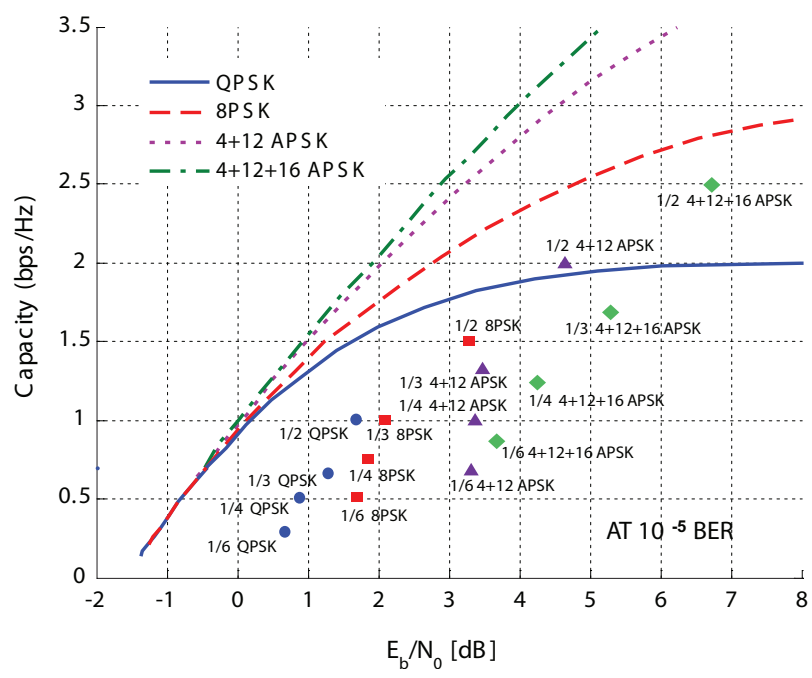

Fig. 5. Capacity for modulation methods and $E_{\mathrm{b}} / N_{0}(\mathrm{~dB})$ required to satisfy the target BER of $10^{-5}$.

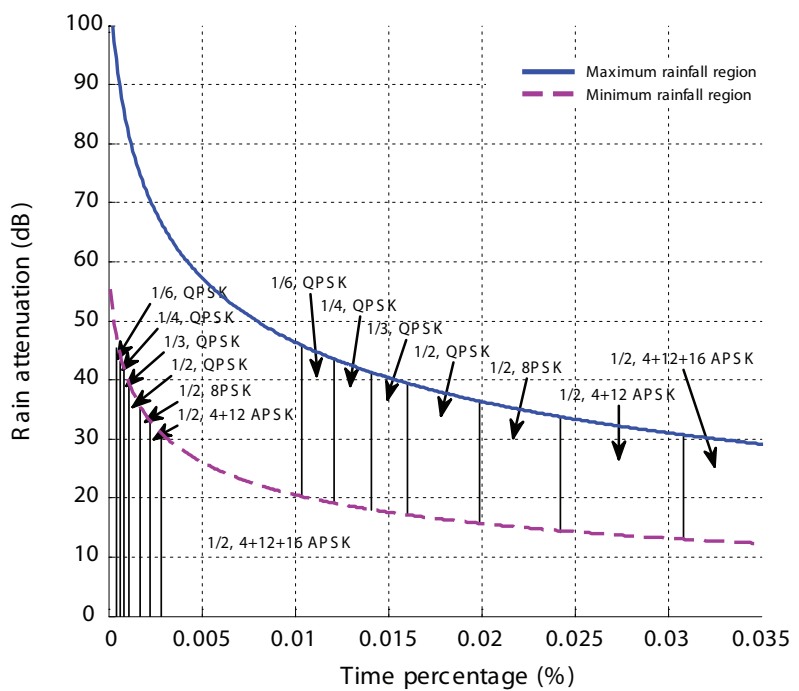

Fig. 6. Rain attenuation and the ACM level for one-year.

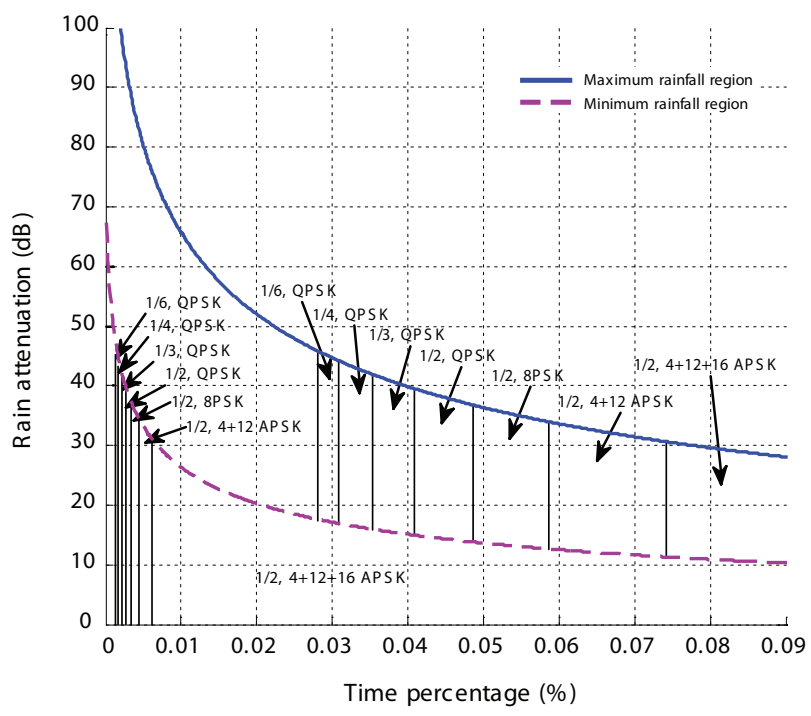

Fig. 7. Rain attenuation and the ACM level for four-month maximum rainfall season.

Table 3. Time percentage for each ACM level.

\begin{tabular}{|c|c|c|c|c|c|c|}
\hline \multirow{2}{*}{ Level } & \multirow{2}{*}{$\begin{array}{l}\text { Coding rate } \\
\text { \& modulation }\end{array}$} & \multirow{2}{*}{$\underset{(\text { dB) }}{\text { Link }}$} & \multicolumn{2}{|c|}{ One-year (\%) } & \multicolumn{2}{|c|}{ Four-month with maximum rainfall (\%) } \\
\hline & & & $\begin{array}{c}\text { Max. rainfall } \\
\text { region }\end{array}$ & $\underset{\text { Min. rainfall }}{\text { region }}$ & $\underset{\text { Max. rainfall }}{\text { region }}$ & $\begin{array}{l}\text { Min. rainfall } \\
\text { region }\end{array}$ \\
\hline 1 & 1/6, QPSK & 45.53 & 0.0106 & $5.223 \times 10^{-4}$ & 0.0286 & $1.563 \times 10^{-3}$ \\
\hline 2 & $1 / 4$, QPSK & 43.50 & 0.0121 & $6.722 \times 10^{-4}$ & 0.0322 & $1.890 \times 10^{-3}$ \\
\hline 3 & $1 / 3$, QPSK & 41.93 & 0.0135 & $8.106 \times 10^{-4}$ & 0.0353 & $2.189 \times 10^{-3}$ \\
\hline 4 & $1 / 2$, QPSK & 39.75 & 0.0157 & $1.046 \times 10^{-3}$ & 0.0403 & $2.683 \times 10^{-3}$ \\
\hline 5 & $1 / 2,8$ PSK & 36.44 & 0.0198 & $1.525 \times 10^{-3}$ & 0.0497 & $3.663 \times 10^{-3}$ \\
\hline 6 & $1 / 2,4+12$ APSK & 33.77 & 0.0242 & $2.076 \times 10^{-3}$ & 0.0594 & $4.729 \times 10^{-3}$ \\
\hline 7 & $1 / 2,4+12+16$ APSK & 30.71 & 0.0308 & $2.912 \times 10^{-3}$ & 0.0738 & $6.385 \times 10^{-3}$ \\
\hline
\end{tabular}

ACM: adaptive coding and modulation, QPSK: quadrature phase shift keying, APSK: amplitude phase shift keying. 
ACM scheme can improve the link availability. However, as shown in Table 2, the spectral efficiencies of level 7 and level 1 are 2.5 and 0.33 , respectively. This indicates that the application of ACM scheme causes a requirement of longer communication time because, as the level of ACM is low, the effective data rate decreases. Hence, the ACM scheme can be used more efficiently when there is sufficient communication time available. In addition, Table 3 represents the time percentage analyzed over one-year period and the four-month maximum rainfall season. However, the available communication time is limited in space communications due to the location of the transmitter and the receiver and/or the movement of heavenly bodies. Thus, rather than applying the results in Table 2 directly, the ACM level analysis provided in this paper should be carried out with the time percentage depending on the available space communication time in practice. If it is assumed that the communications between a lunar probe and a ground station is possible when the moon is seen, averagely 12 hours of communications are possible in one day. Therefore, the time percentage and the link availability for the ACM level provided in this paper can be obtained by considering half of the time percentage shown in Table 3.

\section{CONCLUSIONS}

In this paper, for the reliable telemetry transmission in the space communications in Ka band, we proposed the ACM level to overcome the rain attenuation by considering the CCSDS-recommended turbo codes with the coding rates $1 / 2,1 / 3,1 / 4$ and $1 / 6$, and QPSK, 8PSK, $4+12$ APSK, and $4+12+16$ APSK. In addition, applying the proposed ACM levels to the domestics rain environment, we verified that the time percentage was lowered and the link availability was improved. We expect that the ACM scheme provided here may be a useful reference for designing the space communications system for lunar exploration since it reduces the system power consumption and allows seamless high rate transmission of multimedia telemetry data in Ka band.

\section{ACKNOWLEDGEMENTS}

This research was supported by National Space Lab (NSL) program through the National Research Foundation of Korea funded by the Ministry of Education, Science and Technology (2009-0082437).

\section{REFERENCES}

Consultative Committee for Space Data Systems (CCSDS). Sep 2003, Recommendation for Space Data System Standards: TM Synchronization and Channel Coding, CCSDS 131.0-B-1 Blue Book (Washington, DC: CCSDS Secretariat) [Electronic Document] Available from: http://public.ccsds.org/publications/archive/131x0b1. pdf

Consultative Committee for Space Data Systems (CCSDS). Sep 2007, Recommendation for Space Data System Standards: Flexible Serially Concatenated Convolutional Turbo Codes with Near-Shannon Bound Performance for Telemetry Applications, Experimental Specification CCSDS 131.2-O-1 (Washington, DC: CCSDS Secretariat) [Electronic Document] Available from: http://public.ccsds.org/publications/archive/131x2o1.pdf

Costello, D. J., Jr., Hagenauer, J., Imai, H., \&Wicker, S. B. 1998, ITIT, 44, 2531, doi: 10.1109/18.720548

De Gaudenzi, R., Guillen, A., \& Martinez, A. 2006, IEEE Trans. Wireless Commun., 5, 2396, doi: 10.1109/ TWC.2006.1687763

European Telecommunications Standards Institute (ETSI). 2009, European Standard (Telecommunications Series), ETSI EN 302307 V1.2.1 (2009-08): Digital Video Broadcasting (DVB); Second Generation Framing Structure, Channel Coding and Modulation Systems for Broadcasting, Interactive Services, News Gathering and Other Broadband Satellite Applications (DVB-S2) [Electronic Document] Available from: http://www.etsi.org/deliver/etsi_en/302300_302399/302307/01.02.01_60/ en_302307v010201p.pdf

Lee, H. S. \& Shin, C. H. 1998, JKICS, 23, 1006

Lee, S. R. 2009, KSAS Magazine, 3, 51

Lee, W., Cho, K., Yoon, D., \& Hyun, K. 2010, JASS, 27, 11

Roddy, D. 2006, Satellite Communications, 4th ed. (New York: McGraw-Hill), p.106 